\title{
Surgery watch out the needle eye
}

\author{
Martin Riegler
}

Published online: 16 April 2020

(C) Springer-Verlag GmbH Austria, part of Springer Nature 2020

\section{Dear reader,}

welcome to this special collection (logos) of highly motivated translations of perceptions beautifully composed by the authors of the contributions included in this issue of European Surgery. Logos describes the collection of translations of perceptions and may manifest in any form of human expressions including politics, economy, money, science, art, painting, philosophy, poetry, surgery, radiology, physics, molecular sciences, ethics, morals, history, anatomy and music. Quantum field based poetry (Atemzüge eines Sommertags) forms the beauty of our globe as man questions the essence of all perceptions, which develop at the root of our eye. As such each of you, dear readers, represents the center of its own cosmos and universe. Full stop. Logos became lector after the introduction of the Roman Imperialistic Pressure (RIP)-approach to order the collections based on the assumption: if something helps me to increase power $(€)$ it is right, if it impairs, reduces my power $(€)$ is it wrong. $€$ measures the value and strength of any given power. Any morals, ethics, politics aim to justify any given manifestation of will for power (despotism, industry etc.) by catching us with the universal translator: i.e. money coins might (MCM). Later we will detect that exceptions evolve, when nature interacts with our inventions.

The authors are to be congratulated for the papers, the reviewers are to be thanked for their support; Springer and the industry are to be thanked for offering the publication platform for these collections. Save the phenomena, sozein ta pheinomena! This re-

Doz. Dr. M. Riegler $(\bowtie)$

Reflux \& Health Care, Mariannengasse 10/4/9, 1090 Vienna, Austria

martin.riegler@refluxordination.at lates to the real meaning of sozein and socialism. As a matter of fact, the large majority of socialists have no idea about the real meaning of the word which describes, which aims to describe their political orientation! As such, socialism directly relates and roots within the desire of man to collect (logos) and save (sozein) knowledge. It seems that this quality resides within the inner quality of man. Man saves, man collects and man orders. As such the beauty of science should motivate us to continue the story telling, to continue the myth of man, as described in the recent issues of European Surgery.

The recent issue of European Surgery presented disease. Disease entered the stage and talked to YOU dear readers and explained the way, how disease looks at us, watches us, interprets and translates us into the space time quantum fields of its experience. As such disease told YOU, that most of its expression results from a metabolic response induced by the intake of foods and beverages containing concentrated sugar. And disease explained how that works. In the presence of a specific genetic profile, concentrated sugar induces a neuro-humoral response, which in turn causes the release of proteins, molecules and ions, which in turn act on cells, tissues and fluids, bacteria, viruses and structures and thus orchestrates the large spectrum of symptoms and states of affairs including tumours, inflammations, cancers, diarrhoea, diabetes, hypertension, high levels of cholesterol, obesity, depression, eating disorders etc. Here we continue to follow the presentation of the disease, the stage is open for you, dear disease, please continue to speak to us.

Disease enters the stage and continues to talk:

Thanks for the nice introduction. Now I will continue to explain the second part of my talk and let you know, what concentrated sugar means, 
Fig. 1 The images $(\mathbf{a}-\mathbf{c})$ cartoon the idea of the author showing the interaction between nature (flowers, plants) and inventions of man (concrete, human made structures), as outlined in the text. Images obtained April 1, 2020
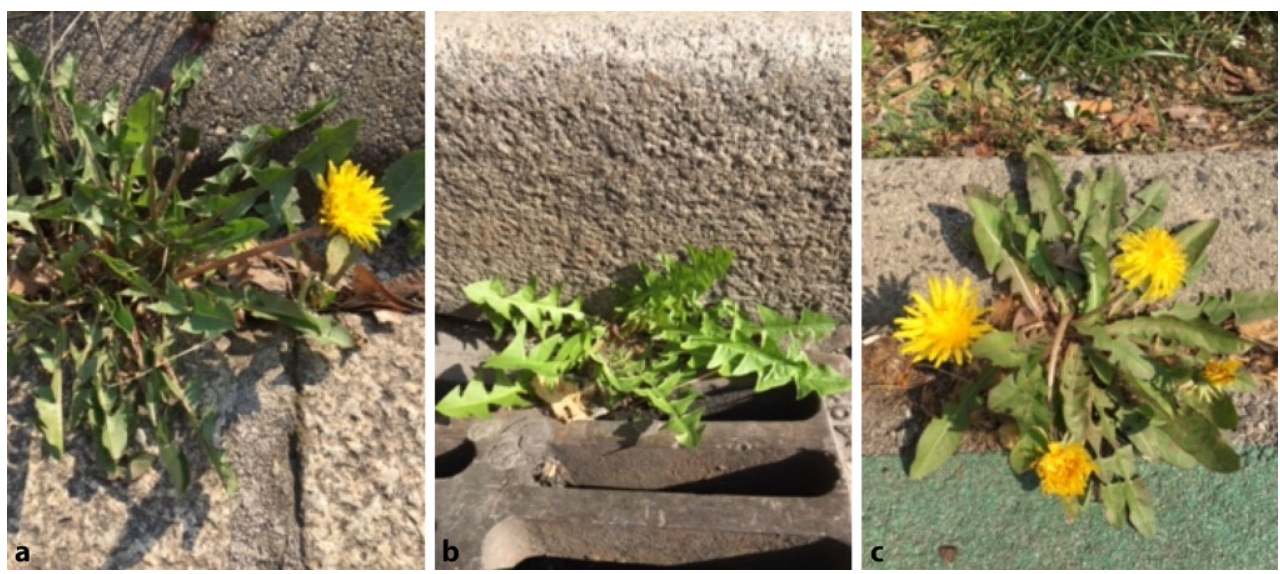

where we find it and what type of foods contain this fundamental type of molecule, which irritates and trembles our metabolism, health and well being.

First of all WE have to understand, YOU have to understand: concentrated sugar represents a special form of space time quantum field orientation, which interacts with the space time quantum fields of your body. And this body (YOUR body, YOUR I and I) continuously waves and interacts with the continuous space time quantum field of the universe (Hubble the telescope!). As such YOU are a special and somehow unique part of the continuous universal space time quantum field, and this space time quantum field follows the segmented regulations of relativity, power, strength and velocity, which in turn are perception - induced phenomena. Without interaction there is no speed, no time, no strength, no power, no will, no fact, no matter, no perception, value and tune. Well taken. Concentrated sugar represents that type of sugar (sweeties, sweeteners, artificial sugar), which is purified as it is taken out of the harmonic orchestration of energy-delivering foods. Sugar is concerted during i.e. cooking, heating a soup, smoothy making etc. In addition life offers germs. For the formation of roots, those germs contain a mixture of proteins (very good) and concentrated sugars (not good for us!). Milk represents a fluid form of germ, i.e. combination of proteins (good) and concentrated sugars (not so good). As thus the history of man teaches that populations and civilisations have been eradicated by the increased consumption of germs, i.e. grain, corn, potatoes, sugar cain, maize, carrot etc. What remained are the architectural remnants of these civilisations and some of their myths, stories and histories survived. Second, starving and hunger hits the metabolism, too. As a consequence, I would recommend you to eliminate foods and beverages containing concentrated sugar and to avoid starving and hunger. Taken together, things get easy when we understand, that I am the imbalanced response following hunger and consumption of concentrated sugar. And here I can end my talk, the rest should happen in your kitchen, in your restaurant, in your bar and in your home land foods and beverages. Do not forget to motivate yourself to follow my recommendations. Finally, I have to admit, that sugar makes the job, but sugar may alter the power and strength of our immune system so that we develop a sensitivity for infections, sugar may foster hyperallergic reactions and responses against special forms of space time quanta. And here I wish to introduce you to my good, old friend, biosheriff Major Role. We know each other since millions of years. Major Role developed out of the preeminent ocean of space time quanta many million years ago, and since that time we collaborated highly successfully. Many forms of diseases result from the interaction between Major Role and the organisms. In addition, specific metabolic diseases foster Major Role induced responses. As such, bio-sheriff Major Role induces and generates a giant neuro-humoral response, which shocks the body, roles the gut, squeezes the skin and floods out the lungs. Due to the current developments on mother earth it is time to introduce to you the next speaker: here comes bio-sheriff Major Role. He will explain us, what happens, if nature interacts with our inventions (Fig. 1).

Major Role, the fascinating and highly effective spheric manifestation of the space time quantum field orchestration, rolls and enters the center of the stage, Major Role waves with his pedicles, which are rooted within the surface of his micro-skin. Electron microscopically scale sized eyes, ears and mouth open their brilliant glances and start to welcome you:

Dear humans! Hi brothers and sisters of the universe! You all come and originate from Africa, I come from the preeminent ocean of emotion 
Fig. 2 The images indicate the consequences resulting from the interaction between nature (Major Role) and human inventions (streets, roads etc.), as outlined in the text. Note the unusual and untypical presence of empty streets during day time. Images obtained in Vienna, Austria during the presence of Major Role (a Vienna Parkring; b Vienna Royal Court theatre, c Vienna Black Mountain Square; d Vienna State Opera). Images obtained April 1, 2020
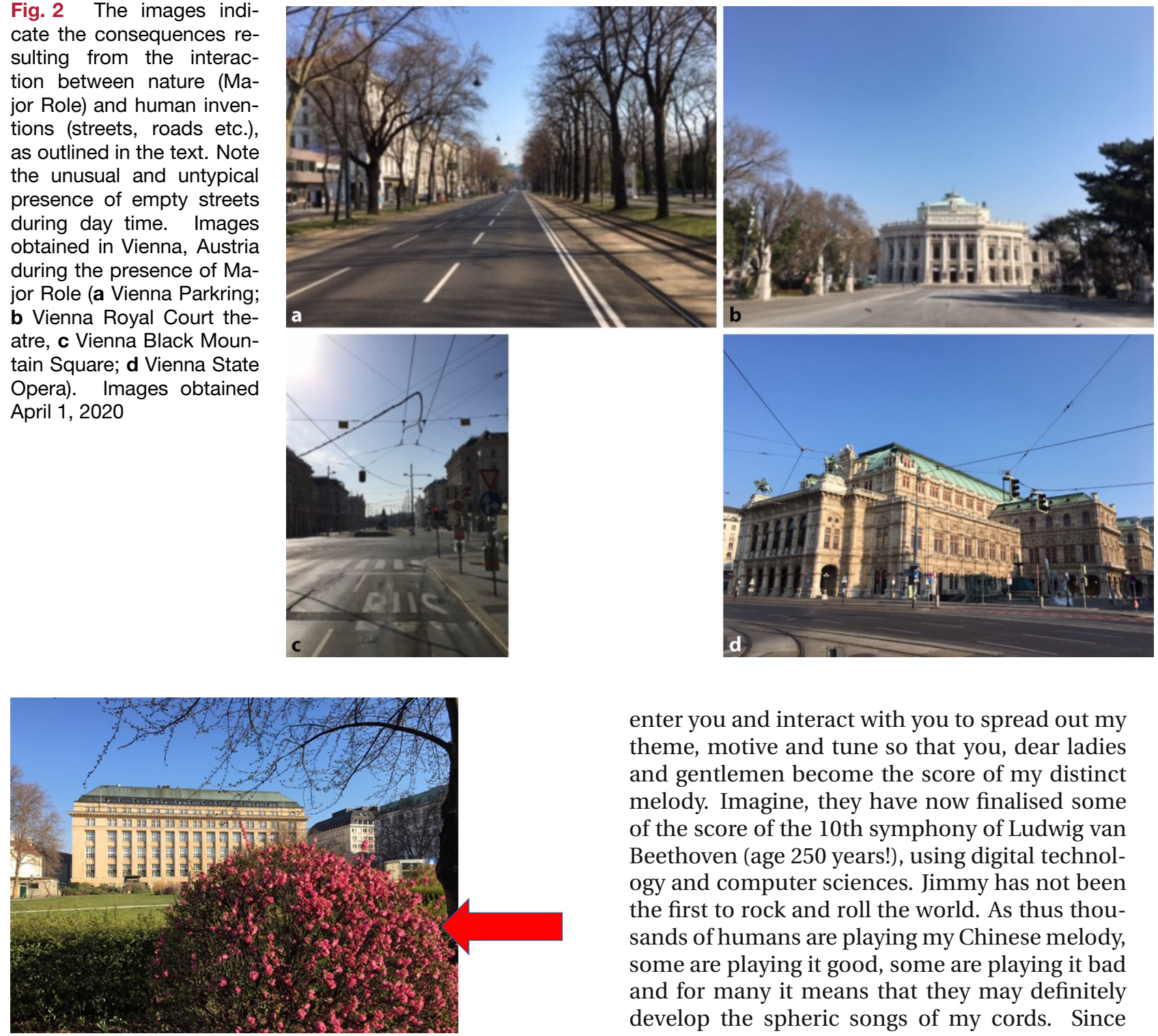

Fig. 3 The image cartoons and models the spheric shape of Major role, as outlined in the text, and mirrors the electron microscopic appearance of Major Role. Note the pedicle-like extrusions (red arrow), which allow Major Role to interact with humans and their inventions, as outlined in the text. The building in the rear depicts the National Austrian Money Hospital adjacent to the old Vienna General Hospital, both are developments and inventions of human mind, nature and thought. In contrast to that, the blue sky evolves due to the interaction between nature and the root of the human eye. Images obtained April 1, 2020

originating at the root of your eye. Wellcome down there. You are a matrix of special order, you form the sheet of a special calculation, you contain mean values and standard deviations of those alive, sick and dead, some of you may follow the guidelines and require, need and precipitate indoor therapy and/or ICU management. My name is, I am, I and I am bio-sheriff Major Role, my spherical geometry allows me to

enter you and interact with you to spread out my theme, motive and tune so that you, dear ladies and gentlemen become the score of my distinct melody. Imagine, they have now finalised some of the score of the 10th symphony of Ludwig van Beethoven (age 250 years!), using digital technology and computer sciences. Jimmy has not been the first to rock and roll the world. As thus thousands of humans are playing my Chinese melody, some are playing it good, some are playing it bad and for many it means that they may definitely develop the spheric songs of my cords. Since I am made in China, where discipline counts over life, I am allowed to "ing form" copy/paste the well reputed inventions and achievements of all Western Societies. I directly belong to nature, and my nature now interacts with the innovations of the human mind, with YOUR inventions and achievements, hopes and desires, fears and thoughts, including economy, money, religion, philosophy, medicine, art, technology, science, publication and journalism, populism, politics, despotism and death. Since your inventions originate from the roots of your brain and the brain belongs to human nature, the products of your mind may also be categorised within the spectrum of natural manifestations. But they have made their way through the spirit, mind and thought of man. So those interactions may be categorised as indirect natural manifestations. It seems there exists no clear answer to that. However, any perceptions represent segmented translations of the universal space time 
quantum field, i.e. energy matters for matter. As a matter of fact, I contribute to alter the surface of the earth, I contribute to alter the appearance of public spaces, roads and cities (Fig. 2). I test the stability of your social, economical, health care and political systems (your mind rooted inventions). This is a great adventure and I have to admit that, as I interact with you (Fig. 3), I will give you a great chance to get rid of me and maybe the interaction between your inventions and me (nature) may stimulate to alter your present and current state of mind. I may demonstrate, that the human civilisation is $v u l$ nerable and soft. As such you may reload and recharge humility, gratitude and thankfulness. Maybe you may stay away from greed, envy, hate and hunger? Maybe I may motivate you to calm down, step back and cool down. Maybe you may redefine your goals? May this translate into the essential meaning of my appearance? May I foster discipline for essence!! Think about it, stay tuned, be you. Never stop your search for the truth underlying your perceptions: WHO $\mathrm{R} U$ ? May the interaction between me and you foster the development of psychological immunity against any form of despotism, uncritical reasoning; and maybe this process may result in effective vaccination against stupidity- and despotism-induced misbehaviour (central maladies vs. Maldives).

Since Major Role harbours no front and rear, no side, upper and lower surface, since Major role is a spheric phenomenon, as assessed by electron microscopy, bio-sheriff Major Role simply shuts down, roles off stage and disappears. The loudspeakers evoke "I shot the sheriff, by Bob Marley". The audience applauses within the matrix of its excel type order of seats and habits. Disease closes the rumor and sessions out over and down:

"Dear madams and sirs, dear unique readers of the Journal, dear all; may the above considerations motivate a re-evaluation of your life. May the review test (everybody talks about testing nowadays!) and address the types, modes and characteristics of your existence and include the provocative aspects and qualities related to foster a novel approach, which of course has to follow the old principles of a-letheia (the uncovered reveals the truth!) and the demands for a new, strong, well structured, accurate language, where the meanings of the terms are clear and reproducible. Vague flaws and inaccurate clouds of semiotics are to be eliminated. This novel approach requires a discipline for an absolute open minded conduct of reasoning and processing of symptoms, signs and qualities. Evaluate yourself and do not run out of your possibilities. May this help you to improve your life quality and to help you to harmonise yourself with your ancestor reflux (i.e. culture), thanks for your attention". Allow yourself to critically evaluate the principles of your existence, social life, politics, economy and your surgery. "Only the repeated accurate proof withstands the test of time".

The session closes and you are the audience. Cancel greed, envy, hate and hunger. Allow and emancipate a united state of mind (alligator shaped Americas). Not all manifestations are to be treated by surgery.

\section{Sincerely,}

\section{Martin Riegler}

Acknowledgements The author thanks the fundamental semiotic contribution related to the ongoing global manifestation of a unique and delicate sound of thunder (Pink Floyd), as related to the role of earth control to Major Role, as outlined in the text (and by the songs of David Bowie). May the text stimulate the reader to critically address and discuss our current form of life, work and reasoning (emancipate yourself from mental slavery, Bob Marley; and "Breaths of a summer day", by Robert Musil).

Conflict of interest M. Riegler declares absence of conflict of interest.

Publisher's Note Springer Nature remains neutral with regard to jurisdictional claims in published maps and institutional affiliations. 